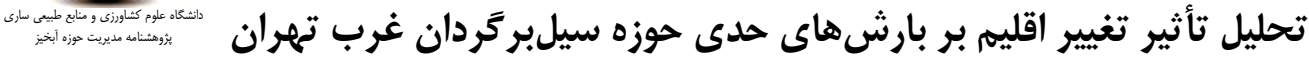

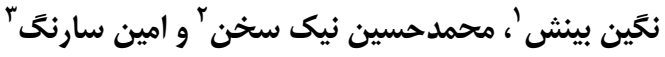

ا و بـ دانشجوى دكترا و استاديار، مهندسى محيط زيستـ منابع آب، دانشكاه تهران

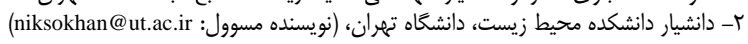

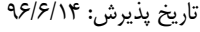

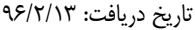

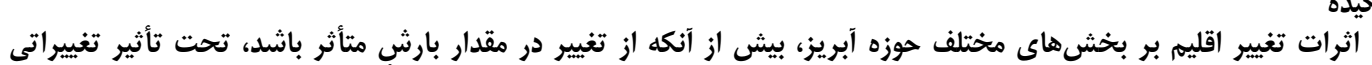

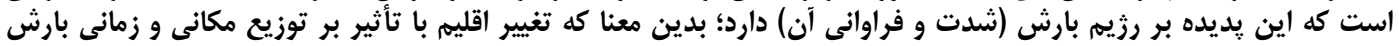

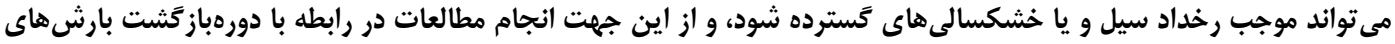

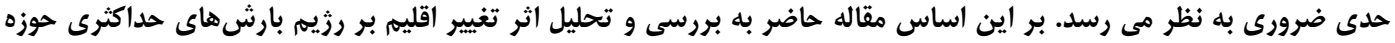

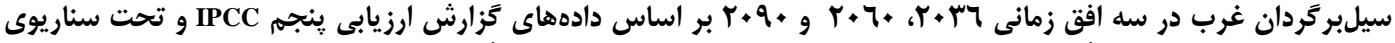

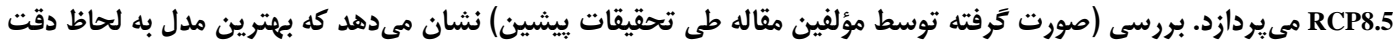

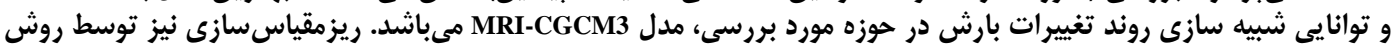

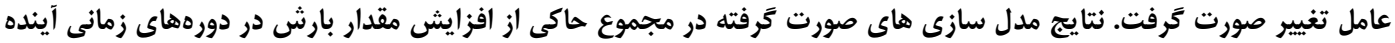

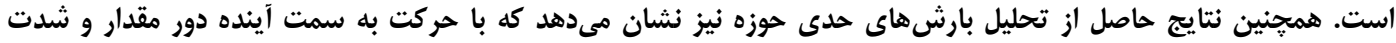

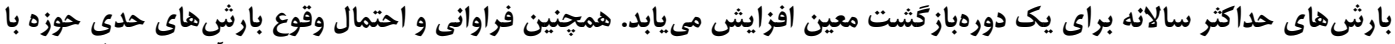

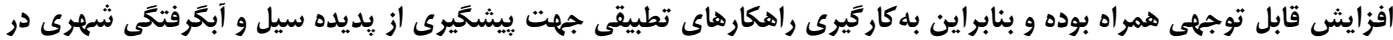

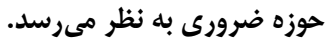

\section{وازههاى كليدى: تغيير اقليم، بارش حداكثر، مدلهاى تردش عمومى جو، دورهبازَّتت، شدت بارش}

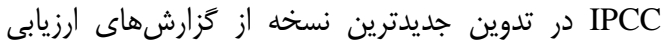

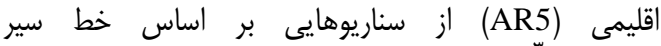

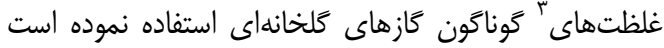

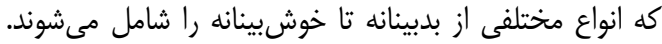

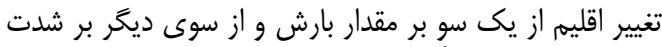

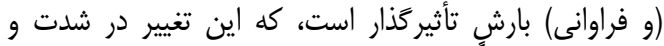

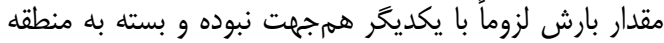

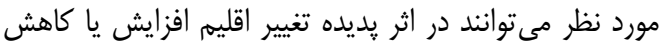

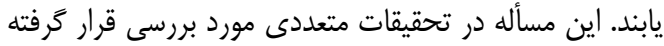

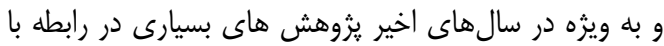

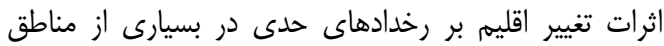

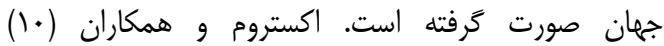
شبيهسازىهايى را براى بارش حداكثر در كشور انغلستان بان إنان

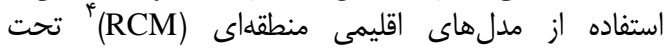

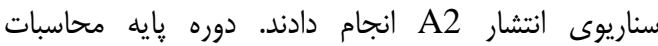

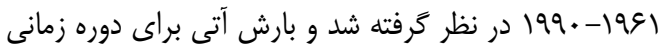

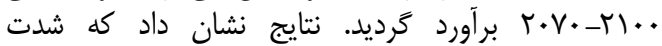

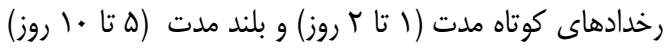

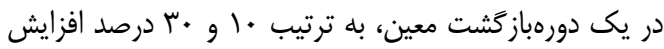

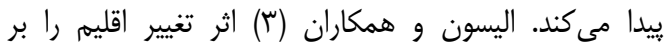

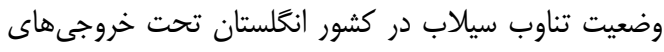

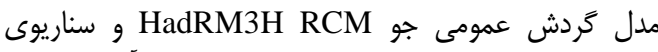

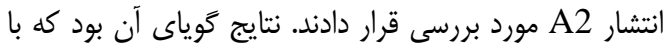

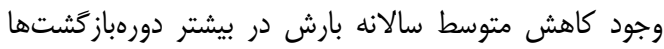

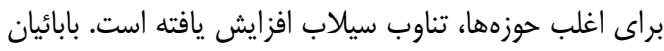

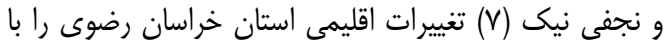
استفاده از خروجى مدل اقليمى تلغيرات التيمى
افزايش مصرف سوختهاى فسيلى در دهاههاى اخير

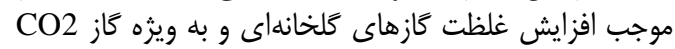

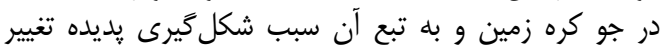

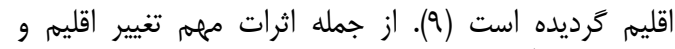

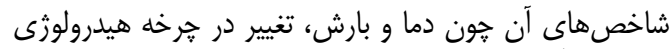

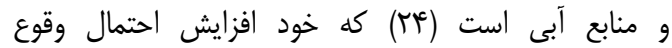

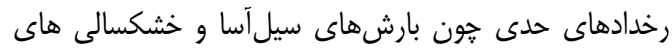

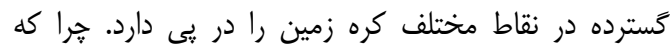

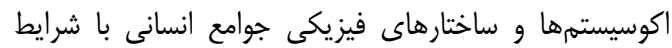

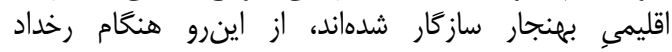

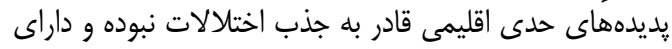

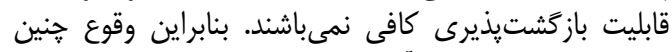

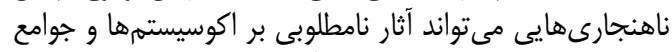

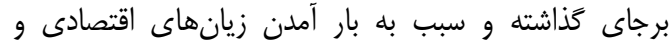

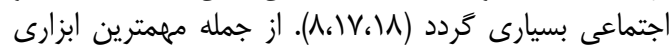

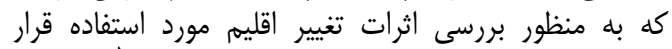

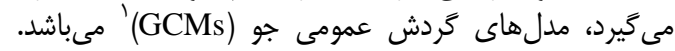

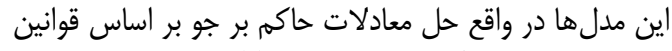

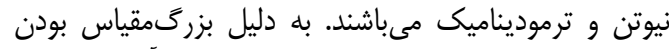

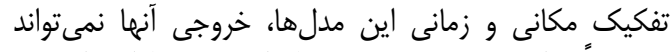

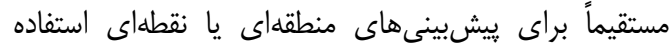

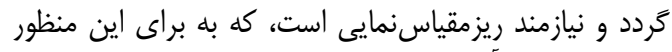

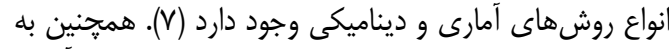

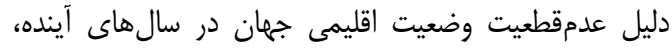

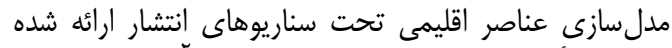

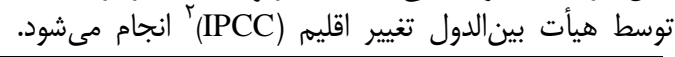

2- Intergovernmental Panel on Climate Change 
مىتواند اثرات جدى بر بخشهاى مختلفى ثون كشاورىى،

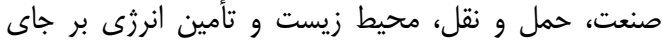

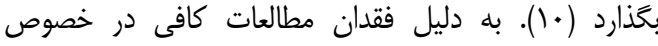

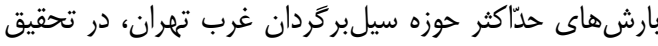

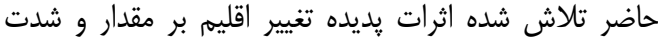

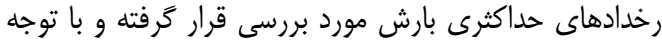

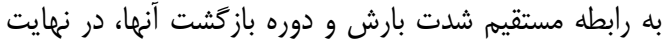

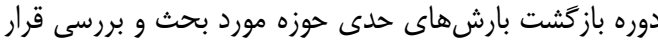

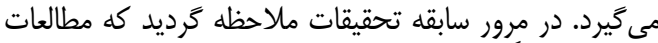

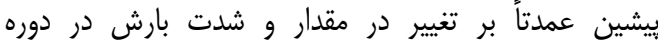

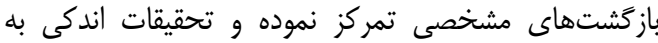

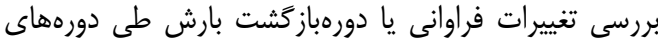

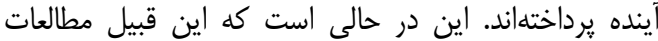

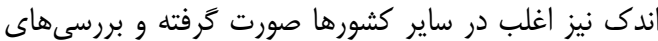

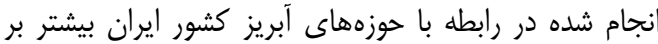

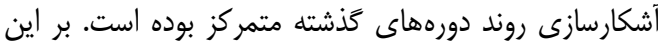

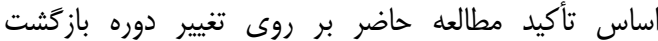

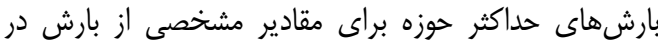

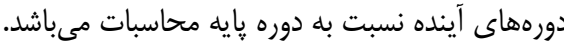

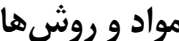 منطقه مطالعاتى}

محدوده مورد بررسى در اين تحقيق، حوزه سيلبركردان

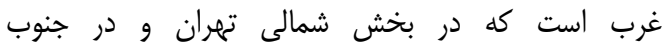

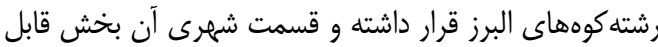

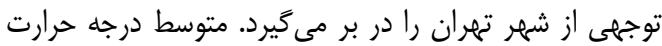

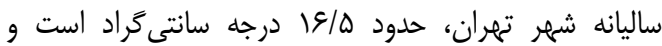

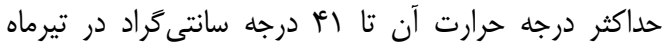

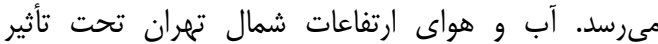

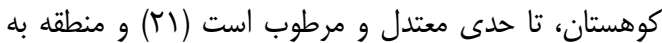

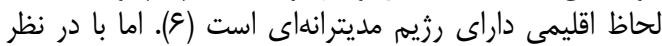

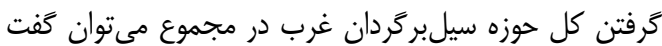

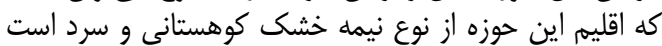

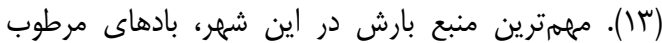

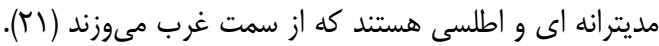

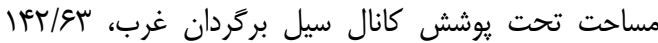

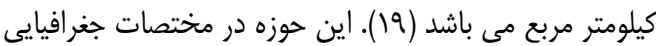

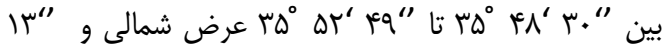
“و $19{ }^{\circ}$

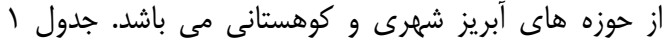

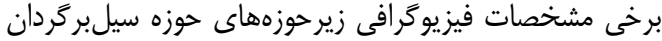
غرب را نشان مى مهدي.

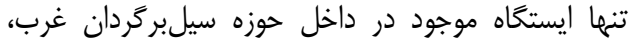

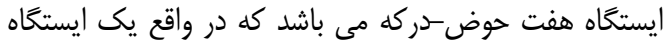

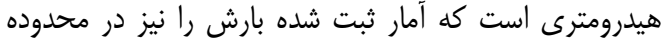

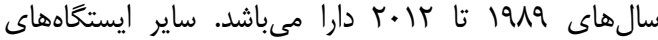

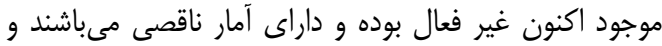

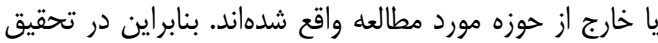
حاضر به منظور افزايش دقت نتايج، از آمار بارش ايستخاه

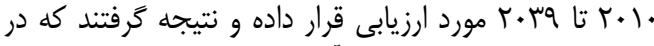

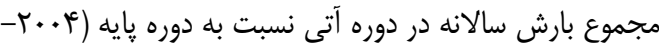

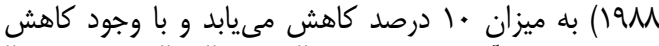

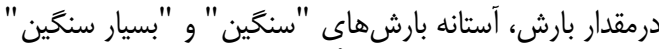

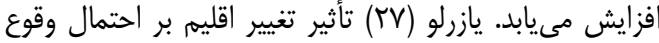

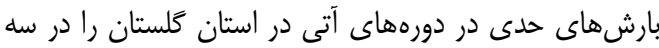

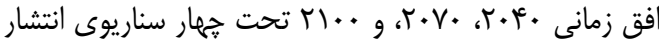

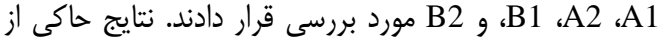

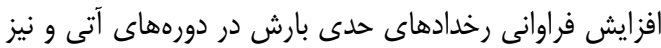

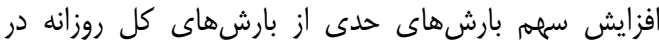

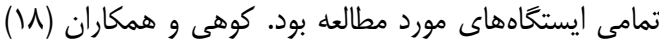

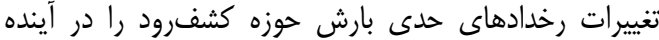

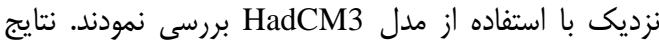

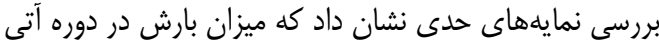

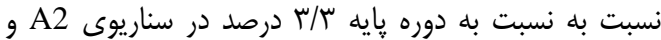

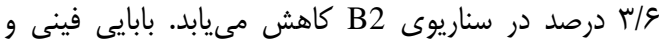

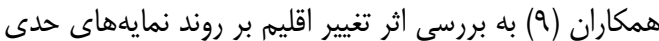

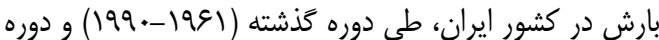

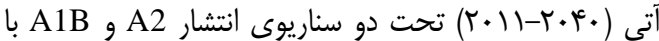

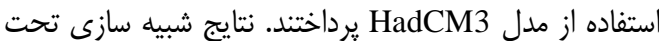

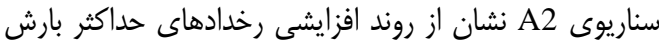

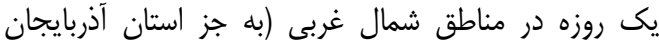

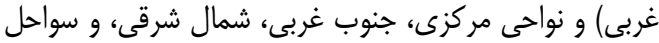

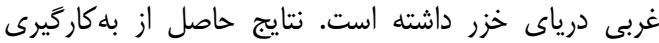

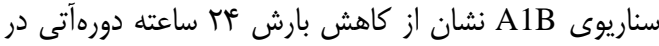

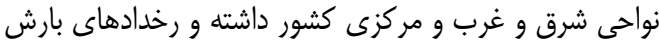

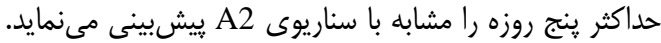

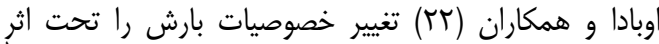

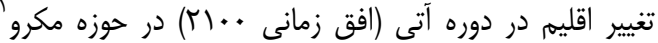

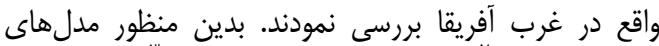

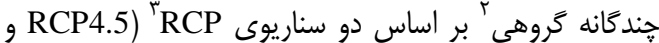

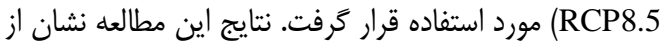

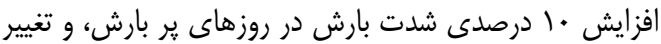

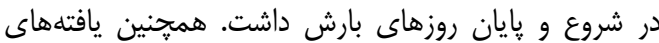

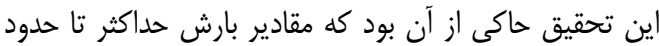

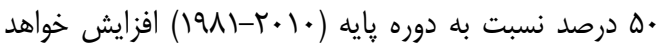

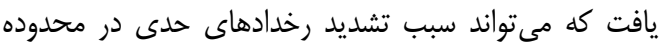

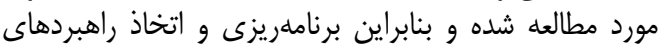

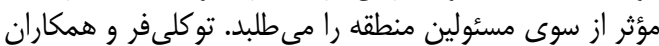

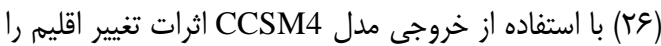

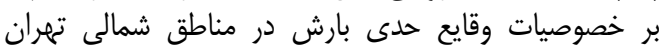

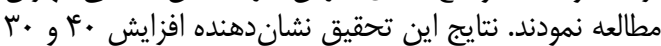

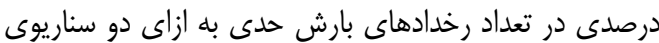

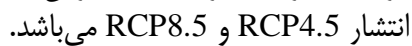

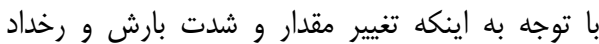

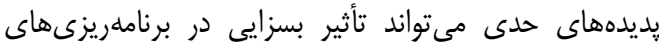

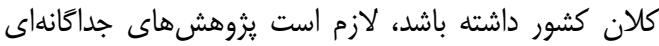

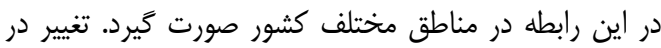

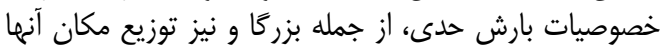




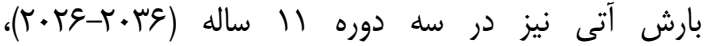

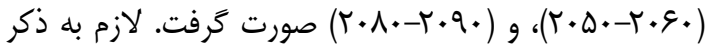

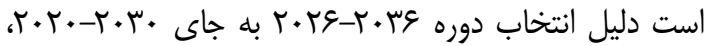

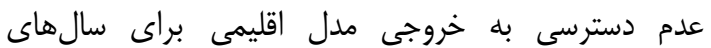

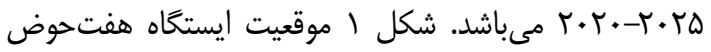

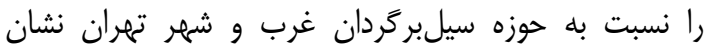

مىدهد.
هفتحوض-دركه كه در داخل خود حوزه مورد بررسى واقع اقدئ

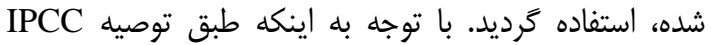

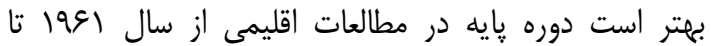

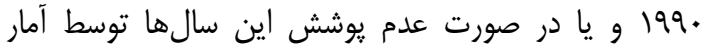

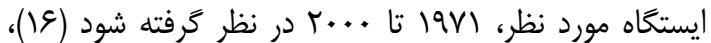

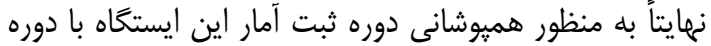

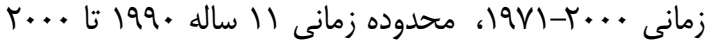
به عنوان دوره يايه محاسبات انتخاب شد و و بررسى تغييرات

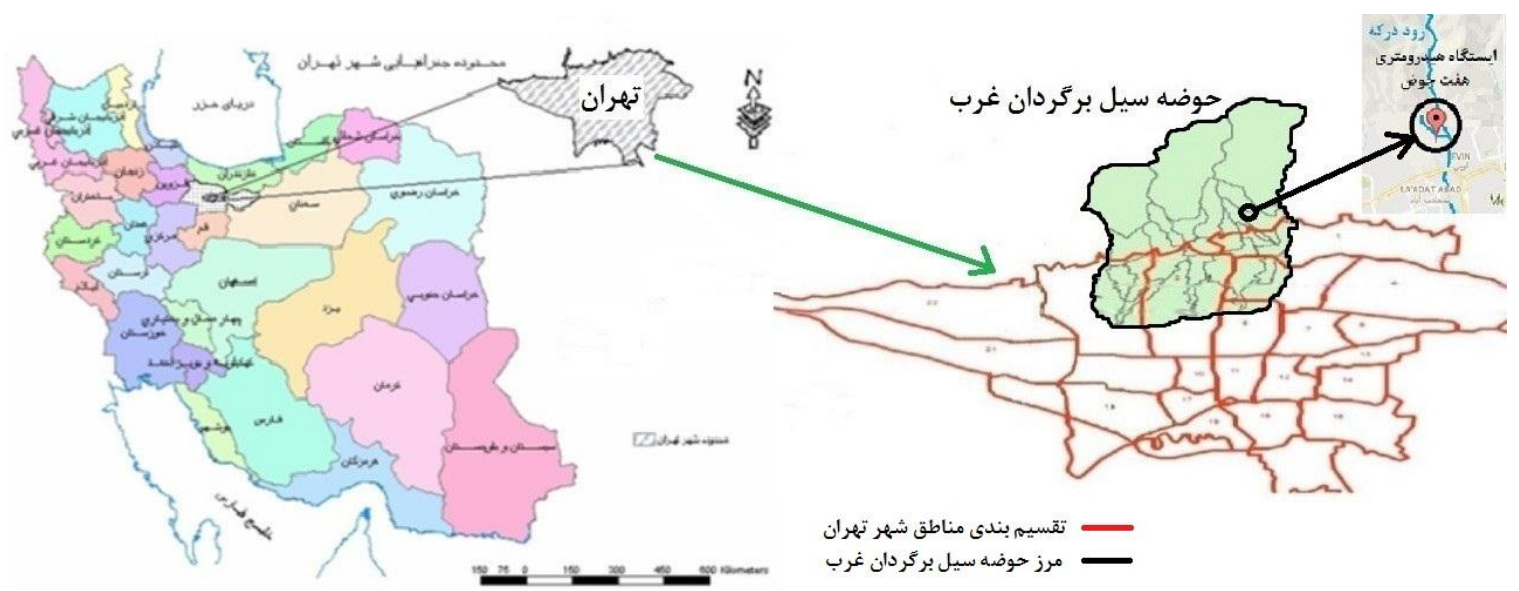

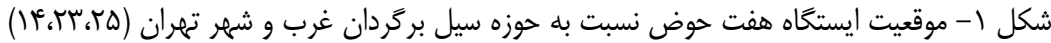

Figure 1. Situation of Haft-Howz station in WFD catchment and city of Tehran (14, 23, 25)

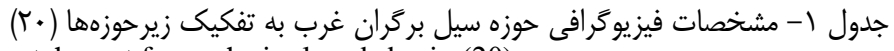
Table 1. Physiographic characteristics of WFD catchment for each single sub-basin (20)

\begin{tabular}{|c|c|c|c|c|c|c|c|}
\hline شيب متوسط & زمان تمركز & $\begin{array}{c}\text { حداكثر ارتفاع) } \\
\text { (متر) }\end{array}$ & $\begin{array}{c}\text { حداقل ارتفاع) } \\
\text { (متر) }\end{array}$ & $\begin{array}{c}\text { طول حوزه } \\
\text { (متر) }\end{array}$ & محيط (تتر) & $\begin{array}{c}\text { (كيلومتر مربع) } \\
\end{array}$ & نام زيرحوزه \\
\hline a. & $r \Delta$ & TVQ. & $1 \Delta \omega$ & $V \cdot \Delta T$ & IVQIS & $1 f / 9$ & حصاركى (بخش كوهستانى) \\
\hline$\Delta / \Gamma$ & $\Delta S$ & lQV. & 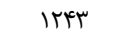 & gNF. & IFVET/V & $9 / 1$ & حصاركى كانال اصلى \\
\hline$V / T$ & $\Delta \Gamma$ & 1910 & שאץו & AFAF & TEQTY & $\mathrm{IV} / \mathrm{A}$ & كانال شاهين-شقايق \\
\hline$\Delta V / T^{c}$ & Fa & re.. & $19 .$. & 99.9 & MEFIT/V & ז & فرخزاد (بخش كوهستانى) \\
\hline $9 / 4$ & $\Delta F$ & MTME & Irs9 & $9 \uparrow \Delta$. & TYMTV/G & $r \cdot / 8$ & فرحزاد كانال اصلى \\
\hline & r. & TQY. & 1090 & $t \cdot t r$ & $91 \% 1 / 4$ & $r / 4$ & كانال مراد آباد \\
\hline & זr & rrq. & I 941 & FMAV & 1. & t & كانال بهرود \\
\hline$\Delta q$ & rq & ґ... & $i v \cdot$. & qTVG & MEITI/A & $r \Delta / \Delta$ & دركه (بخشر \\
\hline $1 \% / T$ & $\Delta \Delta$ & rt.. & . & 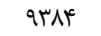 & TMYVT/V & $1 \% / \Delta$ & كانال اصلى \\
\hline V/a & $\Delta F$ & $1 \Lambda .$. & $1 \%+$. & str & $19 \% \mathrm{rq} / 1$ & $1 \% / \Lambda$ & كانال تِه نيزار \\
\hline$\notin / \mathrm{V}$ & 11 & $r \nabla \cdot \cdot$ & 190. & rVa. & $9101 / \Gamma$ & $r / \Lambda$ & كانال خشكه \\
\hline
\end{tabular}

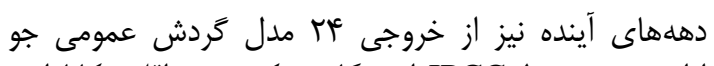

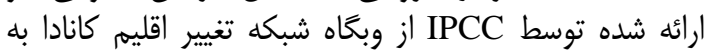

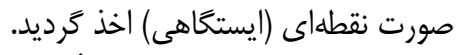

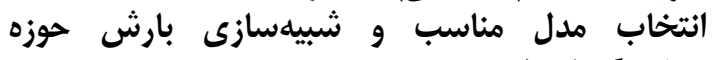
سيلبر مَردان غرب مدل

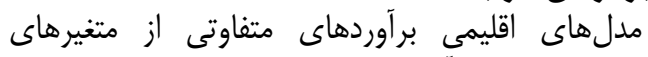

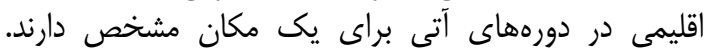

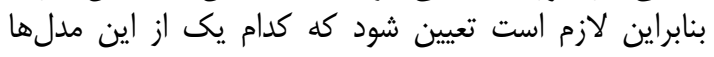

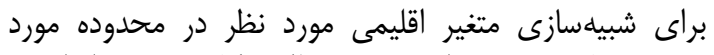

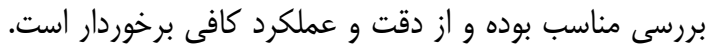

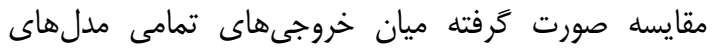

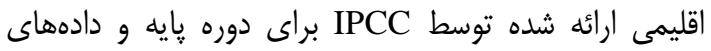

آماده سازى دادهها

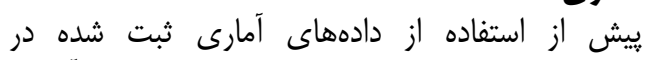

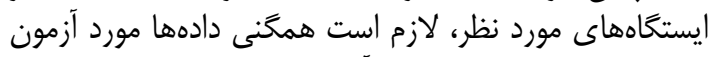

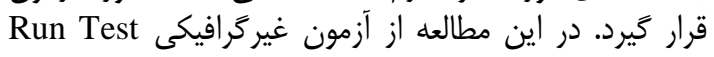

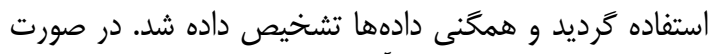

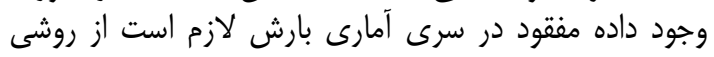

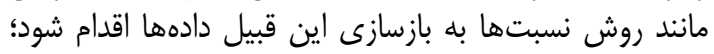

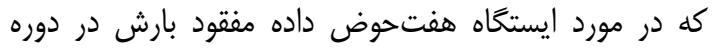

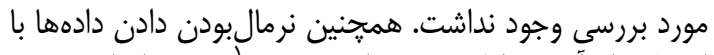

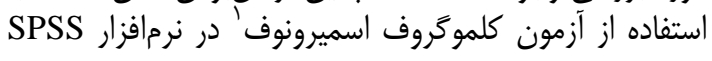

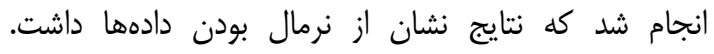

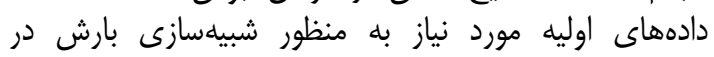


جَّونكى تغيير رزيم بارش حداكثر حوزه مورد بررسى در

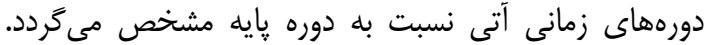

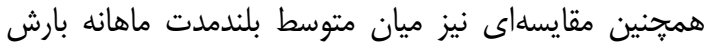

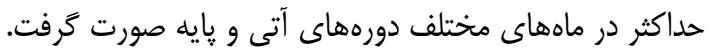

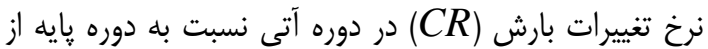

$C R=\frac{\bar{X}_{p}-\bar{X}_{r}}{\bar{X}_{r}} \times 100$ رابطه (ا () محاسبه شد.

كه

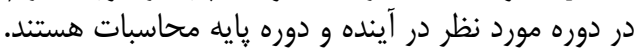

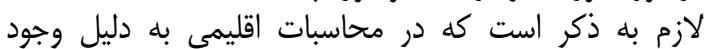

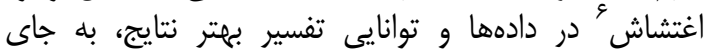

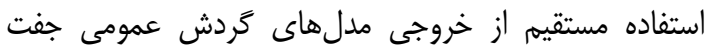

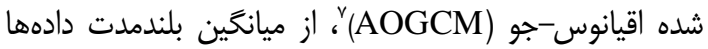

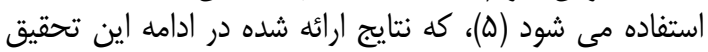
نيز بر همين اساس ارائه كرّديده است.

\section{نتايج و بحث}

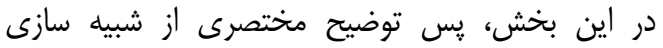

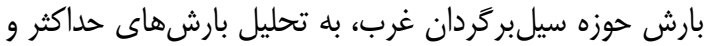

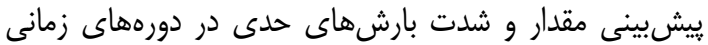
آينده مىيردازيم.

\section{شبيه سازى بارش دورههاى آتى دئ}

مدلسازى بارش دورههاى آينده بر اساس سنار ساريوى آنس انتشار

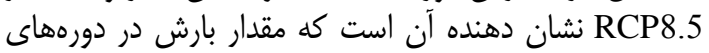

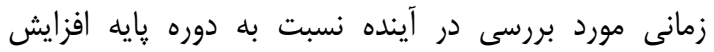

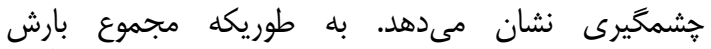

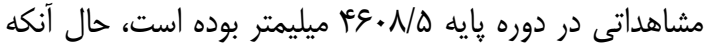

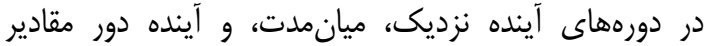

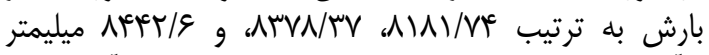

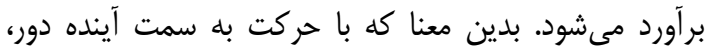

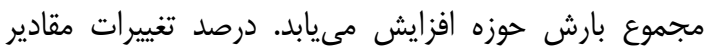

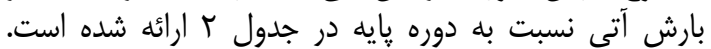

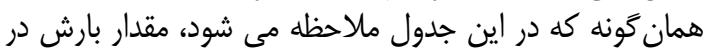

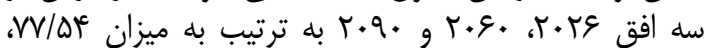

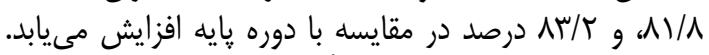

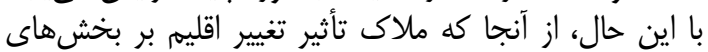

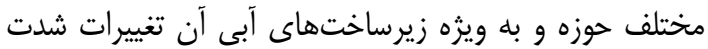

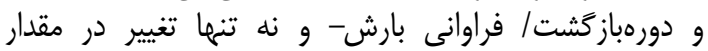

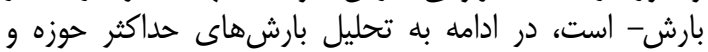

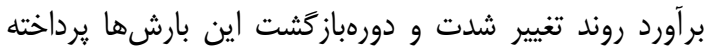

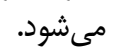

مشاهداتى از طريق سه شاخص جذر ميانگين مربعات خطا

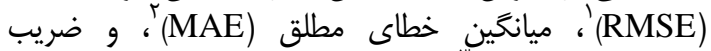
نش-ساتكليف (NS) نشان داد كه مدل MRI-CGCM3

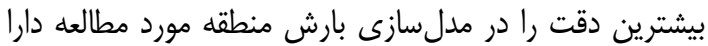

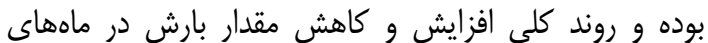

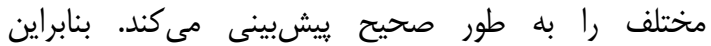

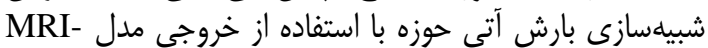
CGCM3

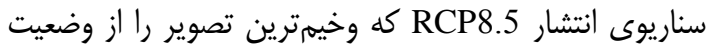

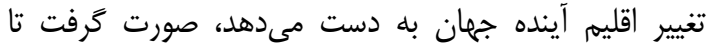

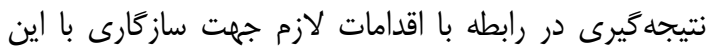

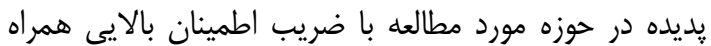
باشد.

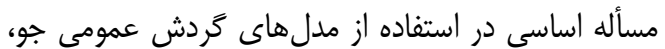

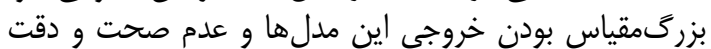

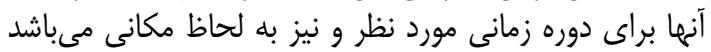

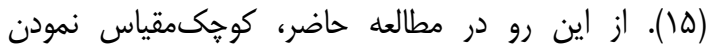

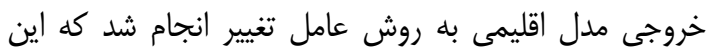

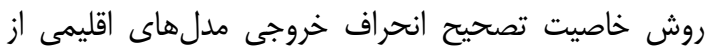
دادههاى مشاهداتى را نيز داراست. تحليل بارش هاى حداكثر إنى

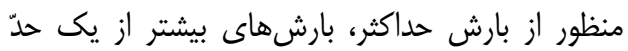

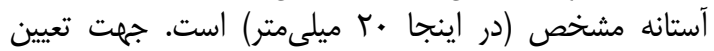

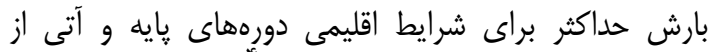

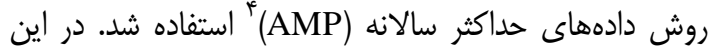

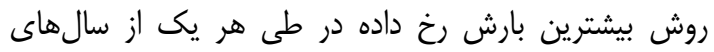

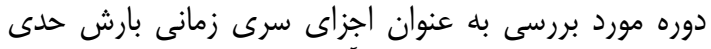

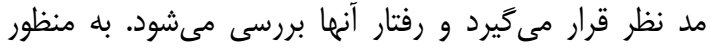

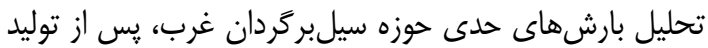

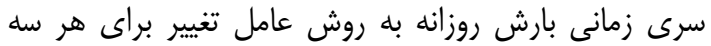

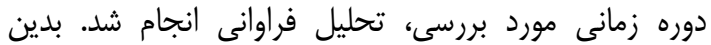

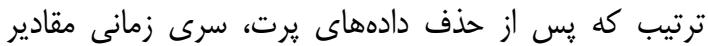

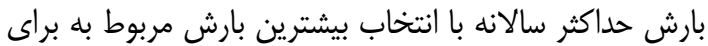

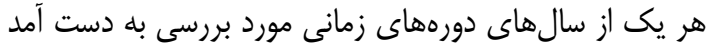

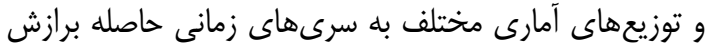

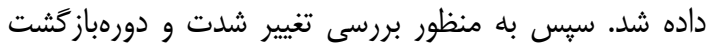

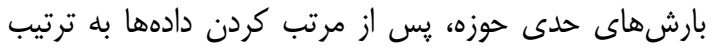

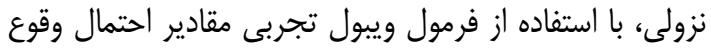

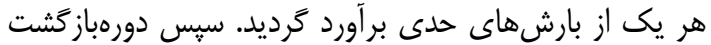

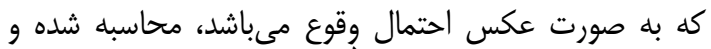

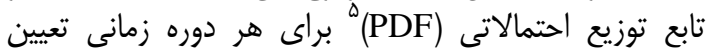

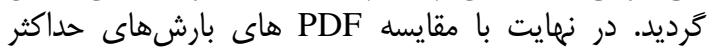
دورههاى آينده با PDF بارشهاى حداكثر دوره مشاهداتى، حاكي،

جدول r- درصد تغييرات مقدار بارش در دورههاى آتى نسبت به دوره يايه تحت سناريوى انتشار RCP8.5 Table 2. Percentage change of precipitation amounts in future periods compared to the baseline under RCP8.5 emission scenario

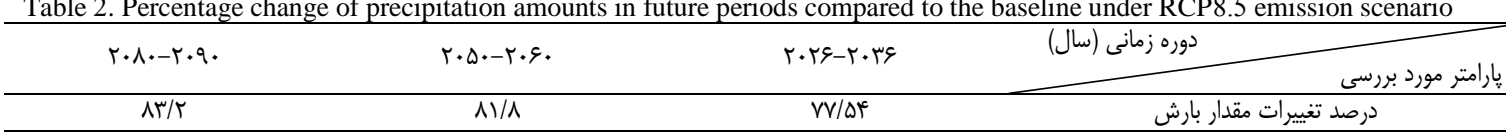


rr.

تا اواسط بهار به وقوع ييوسته كه از اين ميان ماه فوريه در

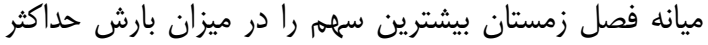

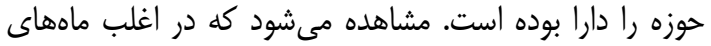

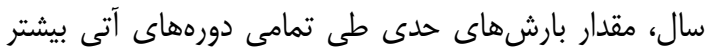
از دوره پايه مىباشد.
تحليل تأثير تغيير اقليم بر بارشهاى حدى حوزه سيلبرگردان غرب تهران

تحليل بارشهاى حداكثر حوزه

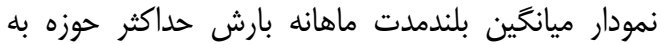

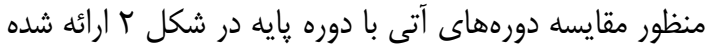

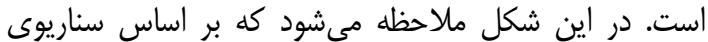
انتشار مورد بررسى، بيشترين مقادير بارش حدى إز از اوايل پإييز

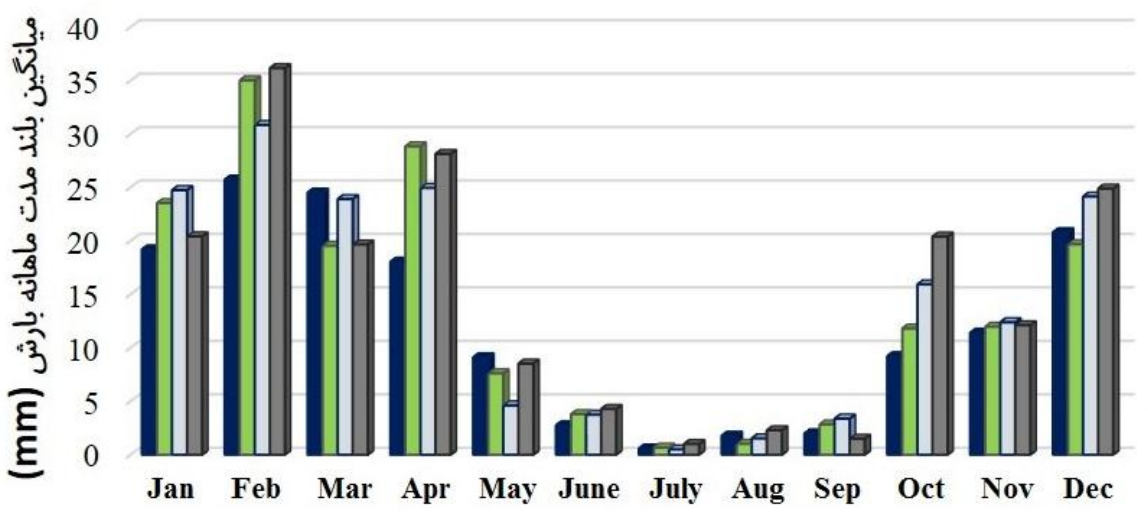

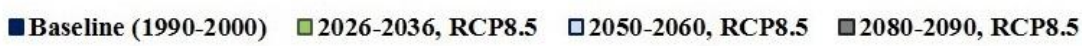

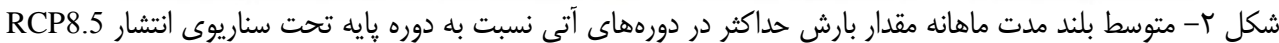

Figure 2. Longterm monthly average of extreme rainfall in future periods compared to the baseline under RCP8.5 scenario

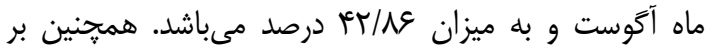

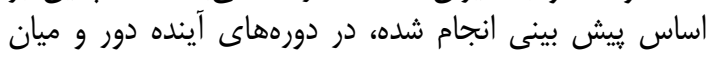

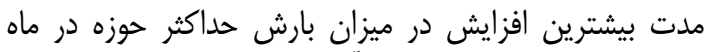

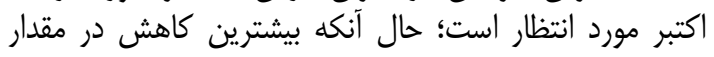

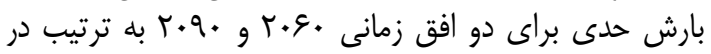

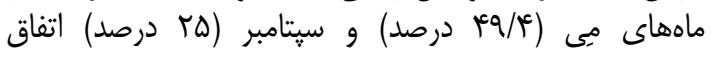
مى مافتد.
محاسبه نرخ تغييرات بلندمدت ماهانه بارش حداكثر حوزه

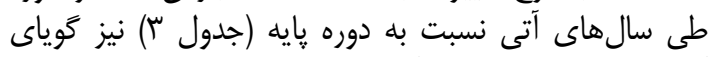

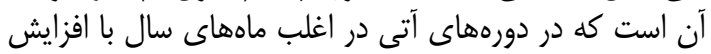

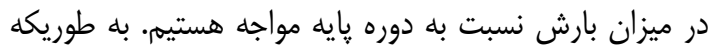

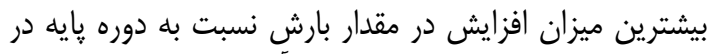

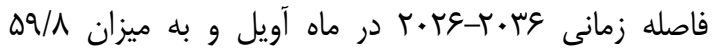

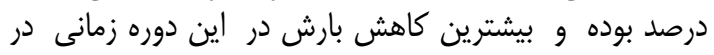

جدول س- درصد تغييرات ميانخين بلندمدت ماهانه حداكثر بارش حوزه طى سه دوره آينده نسبت به دوره پيايه بر اساس سناريوى RCP8.5 Table 3. Percentage change of longterm monthly average of extreme rainfall of the catchment during three future periods compared to the baseline based on RCP8.5 scenario

\begin{tabular}{|c|c|c|c|c|}
\hline \multirow[t]{2}{*}{$r \cdot \Lambda \cdot-r \cdot q \cdot$} & \multirow[t]{2}{*}{$r \cdot \Delta \cdot-r \cdot G \cdot$} & \multirow[t]{2}{*}{$T \cdot r \xi-r \cdot r g$} & \multicolumn{2}{|l|}{ دوره زمانى } \\
\hline & & & & ماههاى سال \\
\hline $9 / \mu \mid$ & TN/AT & $T Y / Q T$ & Jan & \\
\hline$F \cdot / \Delta F$ & $19 / 1 r$ & F & Feb & \\
\hline$-19 / 1$ & $-r / F \Lambda$ & $-r \cdot / r$ & Mar & \\
\hline$\Delta Q / \mathcal{M}$ & TN/TF & $\Delta q / \Lambda$ & Apr & \\
\hline$-\varphi / \Delta \Delta$ & $-r q / 4$ & $-\mid \varepsilon / r V$ & May & \\
\hline$\Delta \Delta / T)$ & $r \Delta / \Delta \Lambda$ & TV/et & Jun & \\
\hline$V \Delta$ & -10 & 10 & Jul & \\
\hline TN/DV & $-14 / r q$ & - FT/NE & Aug & \\
\hline$-r \Delta$ & $99 / 44$ & $|T| / q V$ & Sep & \\
\hline$|r| r \mid$ & $V T / V T$ & $K N \cdot r$ & Oct & \\
\hline $9 / 1$ & $N / \Delta F$ & $F / M$ & Nov & \\
\hline $\mid F / g \psi^{c}$ & $|\varepsilon| \cdot V$ & - & Dec & \\
\hline
\end{tabular}

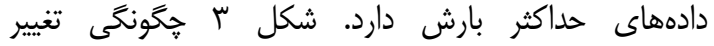

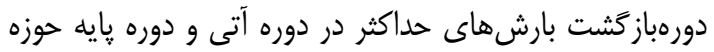

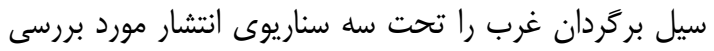

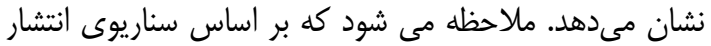

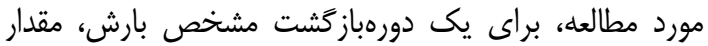

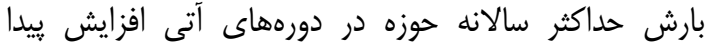

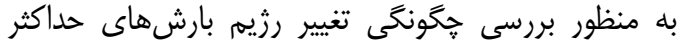

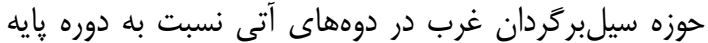

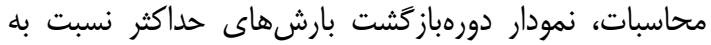

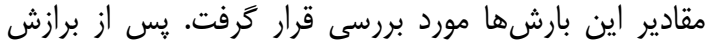

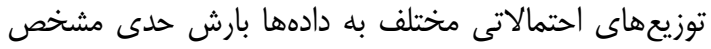

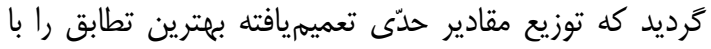


كاهش نشان مىدهد، و اين كاهش دورمباز كشت (افزايش

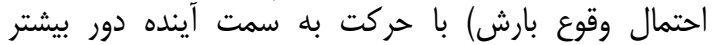
مى شود.
مى كند. به عبارت ديخر احتمال وقوع بارش حدى دور در دوره

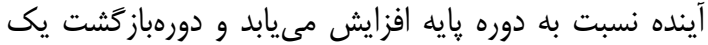
مقدار مشخص از بارش حدى در آينده نسبت بايه دورائ دوره بايه يكايه

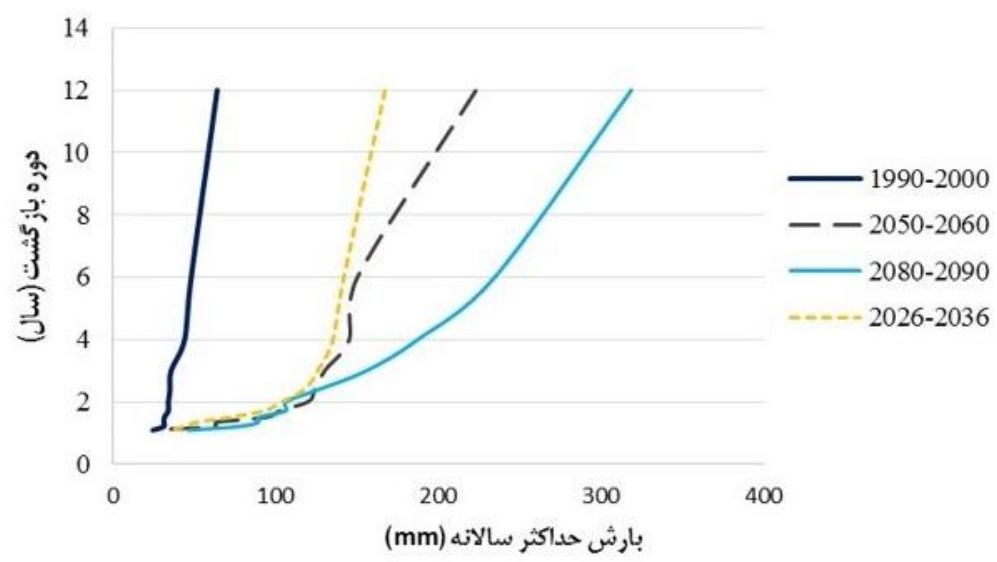

شكل بـ- مقايسه تغييرات دورهباز كشتهاى ميانكين بارش هاى حدى سالانه حوزه در دورههاى آتى و دوره بِايه

Figure 3. Comparison of changes in return periods of average annual extreme rainfalls of the catchment for future and base time periods

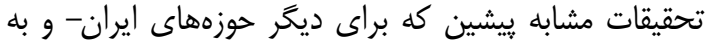

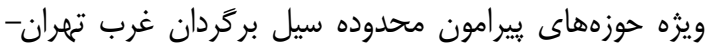

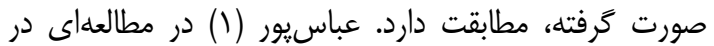

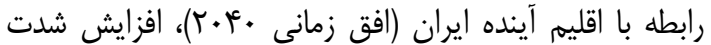

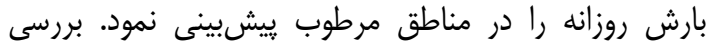

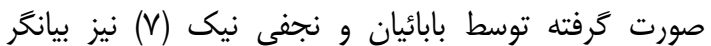

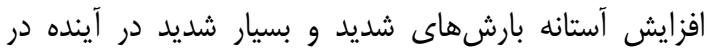

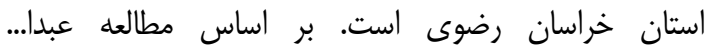

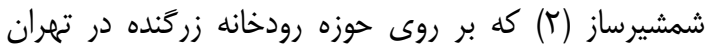

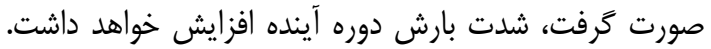

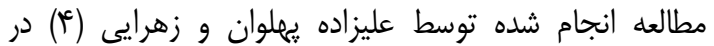

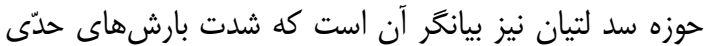
با كذر زمان در حال افزايش است.
به منظور بررسى تغييرات شدت بارش در دورههاى آينده

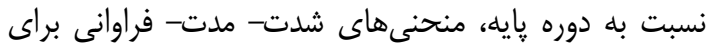

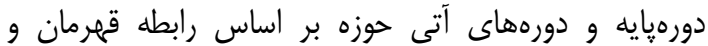

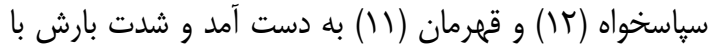

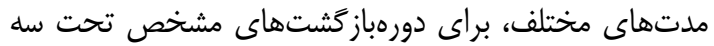

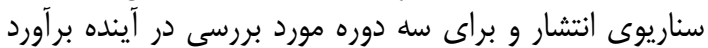

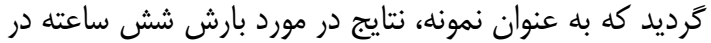

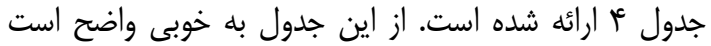

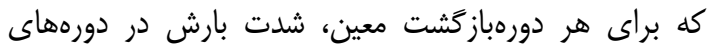

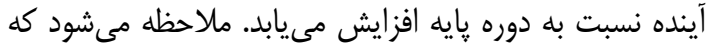

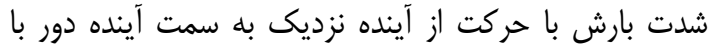

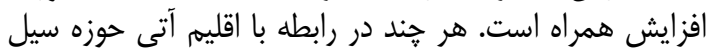

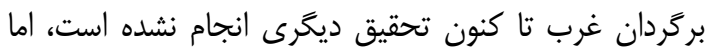

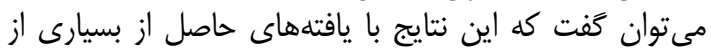

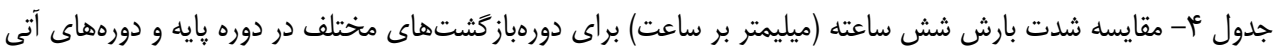
Table 4. Comparison of 6-hour rainfall intensity (m/hr) for different return periods in baseline and future periods

\begin{tabular}{|c|c|c|c|c|}
\hline$r \cdot \Lambda \cdot-r \cdot q \cdot$ & $r \cdot \Delta \cdot-r+\varphi$. & L.te-t.re & 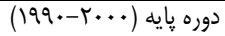 & دورهباز گشت \\
\hline $1 / V \Delta$ & $1 / 99$ & $1 / 99$ & $1 / \Delta 1$ & $r$ \\
\hline$\Delta / F V$ & $\Delta / r q$ & $\Delta / 19$ & $r / N$ & $\Delta$ \\
\hline$V / 94$ & $\mathrm{~V} / \mathrm{GV}$ & V/DT & S/NE & 1. \\
\hline $11 / \cdot \varphi^{f}$ & $1.19 \mathrm{~V}$ & $1 . / 41$ & Q/DT & ro \\
\hline سז/ץו & $I T / M$ & $\mid r / 9 \Delta$ & $11 / \Delta 1$ & Q. \\
\hline$|\omega / 9|$ & $\mid \phi / \cdot V$ & $|f / \lambda|$ & $\mid w / 4 V$ & 1.. \\
\hline
\end{tabular}

MRI-CGCM3 عمومى جو صورت گرفته و در نهايت مدل

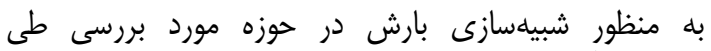

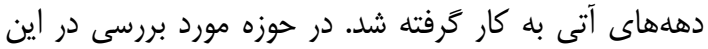

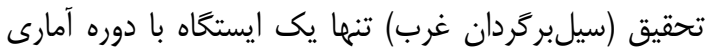

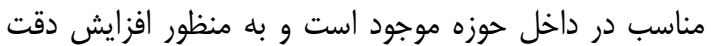

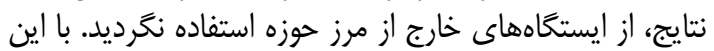

در اين مقاله روند تغييرات بارشهاى حداكثر حوزه سيل

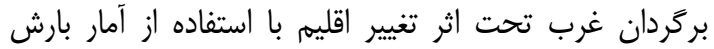

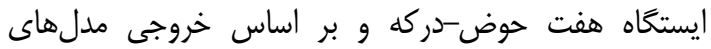

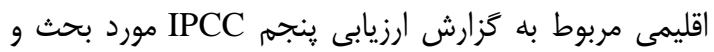

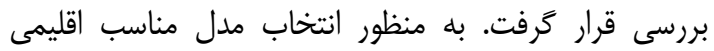

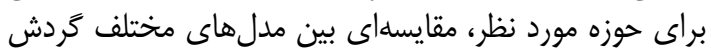


احتمال وقوع و فراوانى بارشهاى حدى حوزه در آينده است.

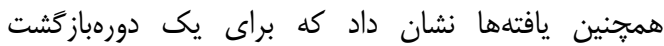

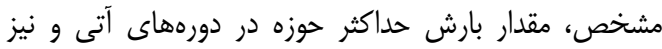

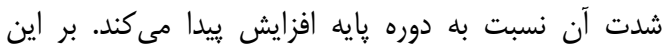

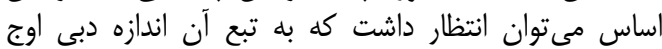

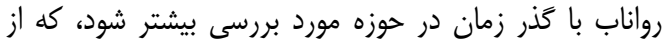

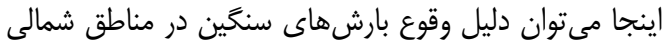

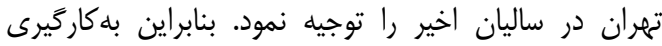

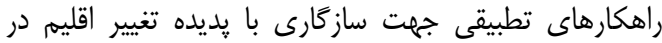

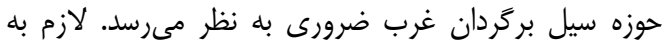

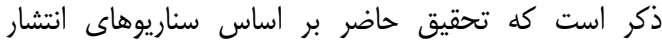

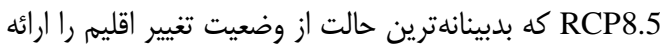

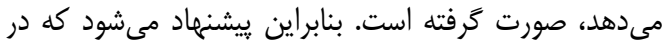

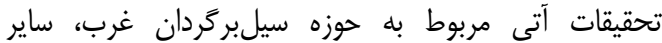

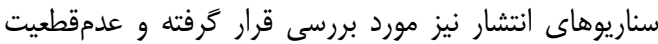
مربوط به سناريوهاى انتشار نيز مورد بررسى و وترار تحليل قرار

$$
\text { كيرد. }
$$

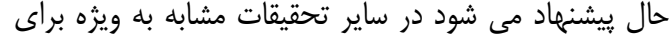

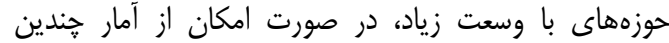

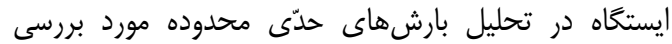

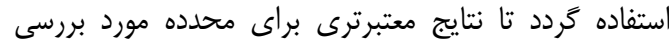

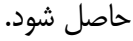

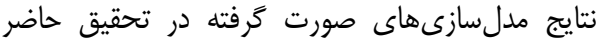

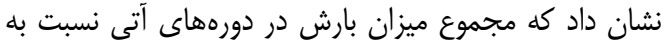

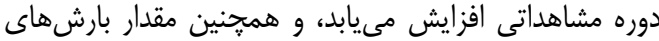

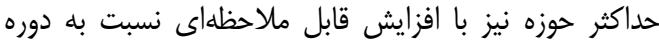

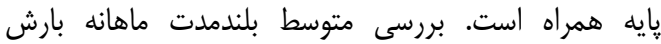

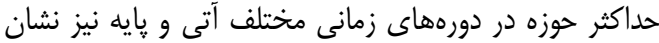

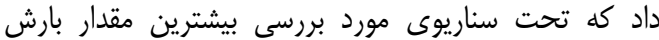

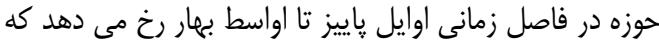

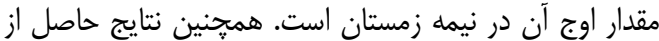

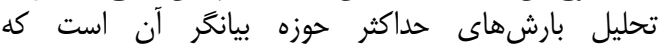

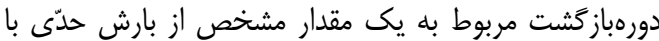
حركت به سمت آينده كاهش مىيابد، كه به معند از بارش افزايش بائ

1. Abbaspour C.K., M. Faramarzi, S. Seyed Ghasemi and H. Yong. 2009. Assessing the impact of climate change on water resources in Iran, Water Research, 45: 1-16.

2. Abdollah Shamshirsaz, Sh., Investigatting the climate change impact on runoff quantity and quality in urban catchment (Case study: Zargandeh channel), M.Sc. Thesis, faculty of Environment, University of Tehran, Tehran, Iran. 2008 [in Persian].

3. Alison, L.K., G.J. Richard and S.R. Nicholas. 2006. RCM rainfall for UK flood frequency estimation. II. Climate change results. Journal of Hydrology, 318: 163-172.

4. Alizadeh Pahlevan, H. and B. Zahraei. 2014. Rainfall statistical downscaling with the aim of evaluating the effects of climate change on extreme events in urban areas, The $1^{\text {st }}$ conference on Climate change and a path to sustainable future, Environment protection agency, Tehran, Iran (In Persian).

5. Ashofteh, P. Sadat, A.R. Massah Bavani. 2009. The effect of climate change uncertainty on temperature and rainfall in Aydoghmoush watershed during 2040-2069, Journal of Knowledge of Water and Soil, 1/19(2): 85-98 (In Persian).

6. Armanshahr Consulting Engineering Company, 2002, Design studies on contour rehabilitation from 1400 to 1800 in $22^{\text {nd }}$ district of Tehran municipality.

7. Baba'eeyan, I. and Z. Najafi Nik. 2010. Analysis of cliamte change in Khorasan-e-Razavi province during 2010-2039 using downscaling the output of GCMs, Journal of Geography and regional development, 8(15): 1-19 (In Persian).

8. Baba'eeyan, I., M. Zarghami, M. Kouhi, A. Baba'eeyan, M. Karimiyan and R. Modirian. 2013. Investigating the behavior of water resources in Ghara-Ghoum catchment under climate change condition (Case Study: Dargaz sub-basin), Journal of Water and Soil (Agricultural science and industry), 27(5): 907-918 (In Persian).

9. Babaei Fini, O.S., A. Ghasemi and A. Fattahi. 2014. Investigating the impact of climate change on trend of extreme rainfall indices in Iran, Journal of Spatial analysis of environmental hazards, 1(3): 85-103 (In Persian).

10. Ekstrom, M., H.J. Fowler, G.G. Kilsby and P.D. Jones. 2005. New estimates of future changes in extreme rainfall across the UK using regional climate model integrations.2. Future estimates and use in impact studies. Journal of Hydrology, 300: 234-251.

11. Ghahreman B. 1996. Updated IDF equation for rainfall in Iran using 1-hour, 10-year rainfall. Journal of Agricultural Science, 6: 13-30 (In Persian).

12. Ghahreman B. and A. Sepaskhah. 1980. Estimating IDF equation for rainfall in Iran using 1-hour, 10year rainfall. $3^{\text {rd }}$ International congress on road and structural engineering, Faculty of Engineering, University of Shiraz, Iran (In Persian).

13. Ghanavati, A., A. Karam and M. Agha Alikhani. 2012. Flood zoning and hazard evaluation in Farahzad basin in Tehran using a fuzy model, Journal of Geography and Environmental Planning, 48(4): 121-142.

14. Google Maps [homepage on the Internet]. Darake River in Tehran, Iran. Available online at: www.maps.google.com.

15. Hosseini, S.H., M.A. Ghorbani, and A. Massah Bavani. 2015. Rainfall-runoff modelling under the climate change condition in order to project future streamflows of Sufichay watershed, Journal of Watershed Management Research, 6(11): 1-14. 
16. IPCC-TGCIA. 1999. Guidelines on the Use of Scenario Data for Climate Impact and Adaptation Assessment. Version 1. Prepared by Carter, T.R., M. Hulme, and M. Lal, Intergovernmental Panel on Climate Change, Task Group on Scenarios for Climate Impact Assessment, $69 \mathrm{pp}$.

17. IPCC. 2007. Climate Change 2007. pp:1-8. In: Solomon, S., D. Qin, M. Manning, Z. Chen, M. Marquis, K.B. Averyt, M.Tignor and H.L. Miller (Eds.), The Physical Science Basis, Contribution of Working Group I to the Fourth Assessment Report of the Intergovernmental Panel on Climate Change, Cambridge University Press, UK.

18. Kouhi, M., M. Mousavi Baygi, A. Farid Hosseini, H. Sanaei Nejad and H. Jabbari Noghabi. 2012. Statistical downscaling and presenting future scenarios of extreme rainfall events in Kashfroud watershed, Journal of climatology research, 3(12): 35-53 (In Persian).

19. Moafi Rabari, A. 2012. Optimal design of WFD (West Flood-Diversion) dimensions based on upland catchment' characteristics, MSc. Thesis, Faculty of Agriculture and Natural Resources, University of Tehran, Iran (In Persian).

20. Moghimi, E. and A. Saffari. 2010. Geomorphological assessment of urban development in the realm of surface drainage basins (Case study: Tehran metropolitan area), Journal of Human science teacher, 14(1): $31 p p$ (In Persian).

21. Nazif, S. 2010. Developing an algorithm for climate change assessment on urban water cycle, $\mathrm{PhD}$ Thesis, Faculty of Engineering, University of Tehran, Iran.

22. Obada, E., E.A. Alamou, J. Zandagba, A. Chabi and A. Afouda. 2017. Change in future rainfall characteristics in the Mekrou catchment (Benin), from an Ensemble of 3 RCMs (MPI-REMO, DMIHIRHAM5 and SMHI-RCA4), Journal of Hydrology, 4(1), 16 pp.

23. Parsa, V. and H. Motiei. 2013. Modelling urban flooding using StormCad (Case study: West floodDiversion and Kan watersheds in Tehran). The $5^{\text {th }}$ conference on Iranian water resource management, Tehran, Iran (In Persian).

24. Pour Hashem, M., B. Bakhtiari and K. Qaderi. 2013. Low flows modelling using temperature and rainfall climatic variables (Case study: Skandari Basin, Esfahan Province), Journal of Watershed Management Research, 4(7): 85-100.

25. Saffari, A. and A. Moghimi. 2009. Geomorphologic assessment of urban development and vulnerability due to land-slide in mountainous hillsides of Tehran, Journal of Natural geography research, 67: 53-71 (In Persian).

26. Tavakolifar, H., E. Shahghasemi and S. Nazif. 2017. Evaluation of climate change impacts on extreme rainfall events characteristics using a synoptic weather typing-based daily precipitation downscaling model, Journal of Water and Climate Change, in press (uncorrected proof), Available online 15 May 2017, 24 pp.

27. Yazerlou, B. 2013. The effect of climate change on occurrence probability of extreme rainfalls in future periods (Case study: Golestan province), Msc. Thesis, Faculty of Water Science Engineering, Gorgan University of Agricultural Science and Natural Resources, Gorgan, Iran (In Persian). 


\title{
Analysis of Climate Change Impact on Extreme Rainfall Events in the West Flood-
} Diversion catchment of Tehran

\author{
Negin Binesh $^{1}$, Mohammad Hossein Niksokhan ${ }^{2}$ and Amin Sarang ${ }^{3}$
}

1 and 3-PhD student and Assistant Professor of Environmental Engineering-Water Resources, University of Tehran 2- Associate Professor, Faculty of Environment, University of Tehran

Received: May 3, 2017 Accepted: September 5, 2017

\begin{abstract}
The impacts of climate Change, more than being influenced by the change in precipitation amount, is affected by the changes imposed by this phenomenon on rainfall regime (intensity and frequency); it means that climate change can cause extensive droughts/floods due to impact on temporal and spatial rainfall distribution, and hence, conducting some studies on return period of extreme rainfalls seems to be necessary. Current paper investigates and analyzes the effect of climate change on extreme regime in WFD catchment at three time horizons (2036, 2060, and 2090) under A1B, A2, and B1 emission scenarios. Investigations (performed by the authors during previous researches) show that the most accurate which is capable of simulating the trend of precipitation variations in the studies catchment, would be MRI-CGCM3 Downscaling was performed using Change Factor (CF) method. The results are representative of reduction in rainfall amount in future time periods, So that the predicted rainfall reduce between 4.12 to 11.69 percent compared to observed baseline precipitation. In addition the result of extreme rainfall analysis show that by moving towards the distant future, the amount of annual extreme rainfalls decrease for a specific return period. However intensity and return period of a specific extreme rainfall increases significantly and therefore, applying adaptive measures for urban flood prevention in the catchment appears to be necessary. Also the probability of extreme rainfall occurrence with a given value in distant future is greater than near future.
\end{abstract}

Keywords: Climate change, extreme rainfall, general circulation models, rainfall intensity, Return periods 